\title{
Efficient Tunable Plasmonic Mode Converters Infiltrated with Nematic Liquid Crystal Layers
}

\author{
Randa H. Kabeel
}

Mansoura University

Nihal F.F. Areed

Mansoura University

Mohamed Hameed

Zewail City of Science and Technology

Salah S. A. Obayya ( $\nabla$ sobayya@zewailcity.edu.eg)

Director of centre for Photonics and Smart Materials-Zewail City of Science and Technology- Sheikh Zayed District, 6th of October City, Giza, Egypt. https://orcid.org/0000-0003-2436-0791

\section{Research Article}

Keywords: Plasmonic, Mode conversion, TM/ TEM modes, Nematic liquid crystal (NLC), Finite element method (FEM)

Posted Date: May 17th, 2021

DOl: https://doi.org/10.21203/rs.3.rs-510999/v1

License: (c) (i) This work is licensed under a Creative Commons Attribution 4.0 International License. Read Full License

Version of Record: A version of this preprint was published at Optical and Quantum Electronics on July 22nd, 2021. See the published version at https://doi.org/10.1007/s11082-021-03086-5. 


\title{
Efficient Tunable Plasmonic Mode Converters Infiltrated with Nematic Liquid Crystal Layers
}

Randa H. Kabeel ${ }^{1}$, Nihal F.F. Areed ${ }^{1}$, Mohamed Farhat O. Hameed ${ }^{2,3,4^{*}}$, Salah S.A. Obayya ${ }^{1,2^{*}}$

${ }^{1}$ Department of Electronics and Communications Engineering, Faculty of Engineering, Mansoura

University, Mansoura35516, Egypt

${ }^{2}$ Centre for Photonics and Smart Materials, Zewail City of Science and Technology, October

Gardens, 6th of October City, 12578, Giza, Egypt

${ }^{3}$ Nanotechnology and Nanoelectronics Engineering Program, Zewail City of Science and

Technology, October Gardens, 6th of October City, 12578, Giza, Egypt

${ }^{4}$ Mathimatics and Engineering Physics Department, Faculty of Engineering, Mansoura University, Mansoura35516, Egypt

Email: mfarahat@zewailcity.edu.eg, sobayya@zewailcity.edu.eg

\begin{abstract}
:
This paper presents two efficient tunable plasmonic mode converters in the infrared regime. The proposed configurations consist of silver layer with etched rectangular holes as metal insulator metal (MIM) waveguides with central cavities. The holes are infiltrated by nematic liquid crystal (NLC) material to increase the transmission through the suggested designs. Additionally, the NLC is used to have tunable operation where the modes at the output port can be controlled. The simulations are carried out using full vectorial finite element (FEM) method. The first design has a single output port which converts the TM mode into the TEM mode with high transmission conversion efficiency of $70 \%$. Further, the second structure allows the generation of the two plasmonic modes simultaneously in two output waveguides. During the biased state, the s- mode transmission conversion efficiency reaches $50 \%$ while the transmission of a- mode at the unbiased state is equal to $49 \%$. It is expected that the proposed tunable mode converters will play an important role in the development of the plasmonic-photonic circuits.
\end{abstract}

Keywords: Plasmonic, Mode conversion, TM/ TEM modes, Nematic liquid crystal (NLC), Finite element method (FEM).

\section{Introduction}

Surface plasmon (SP) modes are excited electromagnetic waves at the metal / dielectric interface. The SP modes take various forms, extended from freely propagating electron density waves over metal surfaces to localized electron oscillations around metallic nanoparticles. The unique properties of the SP modes allow a wide area of practical applications (Zayats et al. 2005; Gramotnev and Bozhevolnyi 2010). In this context, plasmonic biosensors (Hameed et al, 2016; Hameed et al., 2017), and nanoantennas for energy harvesting applications (Obayya et al., 2015) have been reported. The metallic structures have different shapes such as wires (Krasavin and Zayats 2011), V grooves (Moreno et al. 2006; Heikal et al. 2013), wedges (Fernández et al. 2009), nanoantenna (Maier et al. 2002; Dagens et al. 2016) and metal insulator metal (MIM) waveguides (Dionne et al. 2006). The MIM waveguides are the most widely used plasmonic structures due to their unique properties such as strong locality and easy for fabrication. The MIM has been used in a wide range of applications as bend waveguides (Veronis and Fan 2005), optical switches (Tao et al. 2011), demultiplexers (Chen et al. 2015; Wen et al. 2012) and sensors (Lee et al. 2011).

Practically, MIM structures can sustain two plasmonic eigenmodes, termed transvers magnetic (TM) mode and the transvers electromagnetic (TEM) mode, which are also called anti-symmetric mode (a-mode) and symmetric mode (s-mode), respectively. However, each of the SP modes has its unique 
properties. The s- modes have minimum group velocity and better field confinement. Additionally, the amodes can be generated by many configurations such as end-fire excitation and tapered dielectric junctions. However, the s-modes are difficult for excitation because of their antisymmetric lateral field components. The mode conversion between SP modes can be used to solve the problem of s-mode excitation. Further, the mode conversion is the basis for realizing multifunctional nano-circuits. Previous studies show that the mode conversion can be carried out by reshaping phase or redistribution power density of the guided mode. In this regard, the mode conversion has been achieved in silver doublenanowire system (Sun et al. 2013), plasmonic two-wire transmission line (Hung et al. 2012) and MIM waveguides (Wang and Hong 2017; Wang and Yan 2018). The mode conversion can be also realized by transformation media (Kong et al. 2013) or by using selective cavity (Kim et al. 2015). In this regard, Ohana and Levy have introduced mode conversion in nanoscale waveguide using periodic perturbation (Ohana and Levy 2014). Further, Wang and Hong have suggested mode conversion in MIM waveguide using gradient thickness medium (Wang and Hong 2017). Additionally, Wang and Yan have presented mode conversion in MIM waveguide using shifted cavity with maximum transmission of about $35 \%$ at the operating wavelength $\lambda=1.31 \mu \mathrm{m}$ (Wang and Yan 2018). The common drawbacks in the previous studies are the low conversion efficiency and relative fabrication difficulty with no switching capabilities.

Recently, the nematic liquid crystal (NLC) has been endorsed in many photonic devices to control their functions through temperature or external magnetic or electric fields (Hameed et al., 2010). In this paper, two switched configurations for TM/TEM converter are reported with high transmission conversion efficiency. The proposed designs consist of sliver layer with impeded input and output waveguides. Additionally, a central cavity is used to support the mode conversion through the suggested structure. In order to increase the transmission and control the mode conversion, nematic liquid crystal (NLC) of type E7 is infiltrated through the different waveguides. The field propagation and the transmission conversion efficiency are calculated by full vectorial finite element method via COMSOL Multiphysics software package (www.comsol.com). The reported structure with one output port has the ability to change the modes at the output port due to the infiltration of the NLC material. In this context, during the NLC biased state, the a- mode will be converted to the s- mode with high transmission ratio of $70 \%$ which is better than $35 \%$ of the mode converted reported in (Wang and Yan 2018). When the NLC layer is unbiased, the a- mode will propagate along the structure without mode conversion. Further, the second design with two output ports can generate the two plasmonic modes through the two output waveguides. The s- mode transmission conversion efficiency reaches $50 \%$ at the biased state. However, at the unbiased state, the transmission of the a- mode is equal to $49 \%$.

\section{Design Considerations}

In order to understand well the a- mode (TM mode) and s- mode (TEM mode), the conventional MIM structure is introduced in Fig. 1. The conventional metallic structure consists of square waveguide with length of $2 \mu \mathrm{m}$. Further, a cavity layer with thickness $w=800 \mathrm{~nm}$ is used. In this study, silver (Ag) is used with permittivity defined by the Drude mode as given by (Johnson and Christy 1972):

$$
\varepsilon_{m}(\omega)=\varepsilon_{\infty}-\frac{\omega_{p}^{2}}{\omega^{2}+i \omega \gamma}
$$

where the permittivity at infinite angular frequency $\varepsilon_{\infty}$ is set to 3.7, the oscillation damping of electrons $\gamma$ is $2.73 \times 10^{13} \mathrm{rad} / \mathrm{s}$, and the bulk plasma frequency $\omega_{p}$ is $1.38 \times 10^{16} \mathrm{rad} / \mathrm{s}$. The cavity is filtrated with NLC layer of type E7. Equation 2 shows the permittivity of the NLC layer as a function of the rotation angle $\varphi$ of the director of the NLC with the y-axis (Areed et al. 2018; Hameed and Obayya 2011). 


$$
\varepsilon=\left(\begin{array}{ccc}
\mathrm{n}_{\mathrm{o}}^{2} & 0 & 0 \\
0 & \mathrm{n}_{\mathrm{e}}^{2} \cos ^{2}(\varphi)+\mathrm{n}_{\mathrm{o}}^{2} \sin ^{2}(\varphi) & \left(\mathrm{n}_{\mathrm{o}}^{2}-\mathrm{n}_{\mathrm{e}}^{2}\right) \sin (\varphi) \cos (\varphi) \\
0 & \left(\mathrm{n}_{\mathrm{o}}^{2}-\mathrm{n}_{\mathrm{e}}^{2}\right) \sin (\varphi) \cos (\varphi) & \mathrm{n}_{\mathrm{o}}^{2} \cos ^{2}(\varphi)+\mathrm{n}_{\mathrm{e}}^{2} \sin ^{2}(\varphi)
\end{array}\right)
$$

where $\mathrm{n}_{\mathrm{e}}$ and $\mathrm{n}_{\mathrm{o}}$ are the extraordinary and ordinary refractive indices; respectively and can be calculated from the following equation (Li et al. 2005).

$n_{e, o}=A_{e, o}+\frac{B_{e, o}}{\lambda^{2}}+\frac{C_{e, o}}{\lambda^{4}}$

where $\lambda$ is the wavelength and $A_{e}, B_{e}, C_{e}, A_{o}, B_{o}$ and $C_{o}$ are the coefficients of the Cauchy model. The coefficients values at $T=25^{\circ} \mathrm{C}$ are $A_{e}=1.6933, B_{e}=0.0078 \mu \mathrm{m}^{2}, C_{e}=0.0028 \mu \mathrm{m}^{4}, A_{o}=1.4994, B_{o}=$ $0.0070 \mu \mathrm{m}^{2}$ and $C_{o}=0.0004 \mu \mathrm{m}^{4}$. In the presented simulation, the operating wavelength is set to $\lambda=$ $1.31 \mu \mathrm{m}$, and thereby, the values of the $n_{e}, n_{o}$ are equal to 1.698796 and 1.50361 , respectively. Figure 1 (b) and (c) show the captured magnetic field of the a-mode (TM mode) and s-mode (TEM mode) in the propagation ZY-plane at the operating wavelength $1.31 \mu \mathrm{m}$; respectively.

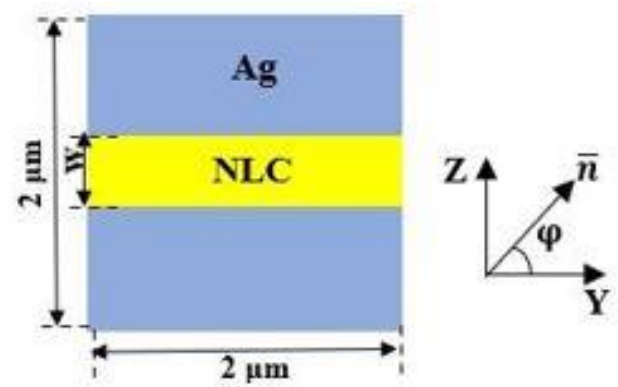

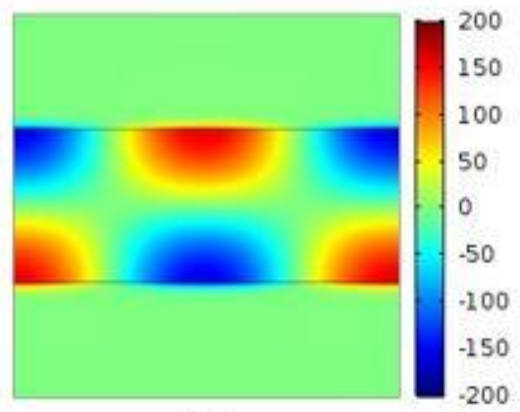

(b)

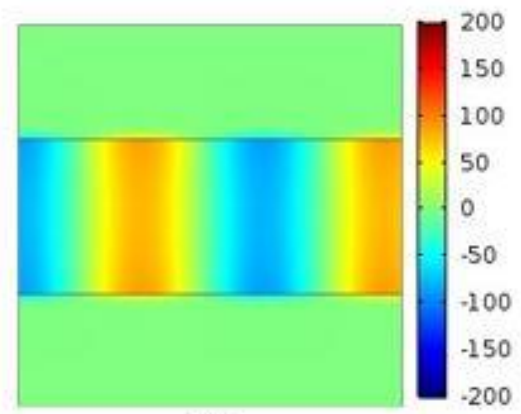

(c)

Fig. 1: (a) The conventional MIM waveguide, and the magnetic field distributions in the $\mathrm{YZ}$ propagation plane of the (b) a-mode (TM mode), and (c) s-mode (TEM mode).

It is believed that the infiltration of the NLC in the cavity with a thickness of $800 \mathrm{~nm}$ can be performed experimentally. Additionally, the proposed in plane alignment of the NLC can be exhibited under the influence of an appropriate homeotropic anchoring conditions (Ren et al. 2008) and under a strong field limit (Wei et al. 2009; Haakestad et al. 2005). In addition, the electric field direction can be controlled using sets of electrodes (Wei et al. 2009) under an effective driving voltage of $50 \mathrm{Vrms}$. The NLC in-plan alignment has been achieved experimentally though hole diameter of $(0.7-1.0 \mu \mathrm{m})$ (Woliñski et al. 2005). Further, the NLC orientation has been successfully achieved in etched silicon on insulator cavity with different 
thicknesses from $400 \mathrm{~nm}$ to $10 \mu \mathrm{m}$ (Desmet and Baets 2006). The applied field is usually driven by a square wave with amplitude normally varying from 0 volt up to 40 volt and frequency varying from $50 \mathrm{~Hz}$ up to $1 \mathrm{kHz}$. A strong field can readjust an NLC rather rapidly, within $100 \mathrm{~ns}$. Additionally, doping the NLC layer by isotropic layers like zinc oxide nano-particles may be useful for decreasing the amplitude of the required voltage (El-Rabiaey et al. 2016; Eskalen et al. 2015). It is also worth noting that the speed of the NLC switching can be increased electrically by modifying the order parameter (EMOP) using ultra-short voltage pulses. For example, thin CCN-47 cells with $5 \mu \mathrm{m}$ thickness can be used to switch NLC using the EMOP effect which allows ultra-fast response times below $30 \mathrm{~ns}$ only. Therefore, mode conversion between modes will not be affected by on or off times (Borshch et al. 2013).

\section{Numerical Results}

\subsection{Tunable TM/ TEM Converter using One Output Waveguide}

The proposed structure consists of a rectangular waveguide with dimensions of $9.1 \mu \mathrm{m}$ and $6.5 \mu \mathrm{m}$. The suggested configuration is based on the mode converter reported in (Wang and Yan 2018) with silver (Ag) metallic layer. Three rectangular holes with thickness $\mathrm{w}=800 \mathrm{~nm}$ are etched in the silver layer to obtain input waveguide, output waveguide and shifted central cavity. The distance between the central cavity and input/ output waveguides are equal to D. Further, the length of the central cavity is $\mathrm{L}=5 \mu \mathrm{m}$ which is selected carefully in order to achieve switched plasmonic mode conversion. The central cavity is shifted by a distance $\mathrm{C}$ from the input waveguide. To allow high transmission and fabrication simplicity and inspired by the geometries suggested by (Wang and Yan 2018), the cavity and the output waveguide are shifted up by $\mathrm{C}=500 \mathrm{~nm}$. Additionally, the distance between the cavity and the input and output waveguide is set to $10 \mathrm{~nm}$, where the thickness, w is taken by $800 \mathrm{~nm}$. It is worth noting that a gap distance of $10 \mathrm{~nm}$ has been experimentally achieved as reported by Siegfried et al. (2011). The holes are infiltrated by NLC layer of type E7 as shown in Fig. 2. (a). The molecules of the NLC at the unbiased state are aligned along the y-axis $\left(\varphi=0^{\circ}\right)$. However, the NLC molecules at the heavily biasing state will be oriented along the $\mathrm{z}$-axis $\left(\varphi=90^{\circ}\right)$. To control the orientation of the NLC molecules, two electrodes are used as shown in Fig. 2(c). In order to examine the electric field distribution along the NLC layer at the heavily biasing state, the Laplace equation $\nabla .(\varepsilon(\mathrm{y}, \mathrm{z}) \mathrm{E})=0$ is solved numerically where $\mathrm{E}$ is the electric field vector and $\varepsilon(y, z)$ is the dielectric constant. The calculated electric field distribution is shown in Fig. 2 (c). It could be noted from the figure that the lines of the electric field are uniformly distributed parallel to the z-axis at the biasing state of NLC where $\varphi=90^{\circ}$.

In the proposed design, the mode conversion is based on adjusting the length of the shifted cavity L that can select the plasmonic mode (a- or s-mode) at the Fabry-Perot (FP) resonance. At the heavy biased NLC, the shifted cavity length, L is adjusted to follow Eq. 4 (Wang and Yan 2018) to allow the conversion from the a- mode to s- mode through the cavity and comes out from the output waveguide.

$\frac{2 \pi}{\lambda} \operatorname{Re}\left(n_{e f f}\right) L+\theta=m \pi$

where, $\operatorname{Re}\left(n_{e f f}\right)$ is the real part of the effective index of the selected mode in the cavity, $m$ is the order of the FP resonance mode and $\theta$ is an additional phase shift due to the reflections inside the cavity. In the unbiased NLC, the FP resonance of the shifted cavity occurs for the a- mode. Thereby, when the input waveguide is excited by the a- mode, it will propagate and will be coupled to the shifted cavity and hence to the output waveguide. However, at the biased state of NLC, the power is transferred from the a- mode 
to the s- mode through the shifted cavity. In this case, the s- mode will propagate and come out from the output waveguide.

Under applying the electric field, the molecules of the NLC layer will be oriented in the same direction of the electric field. The obtained phase shift due to the propagation through the NLC is given by (Eskalen et al. 2015):

$\delta \emptyset=\frac{2 \pi}{\lambda} \Delta n L$

where $\delta \varnothing$ is the total phase shift, $\Delta n$ is the birefringence which is the difference between $n_{e}, n_{o}$ and L is the length of the NLC. It may be seen that the phase shift through the NLC depends on the length L of the NLC layer. Therefore, the distance from the $\mathrm{z}$ - axis will not affect the produced phase shift.
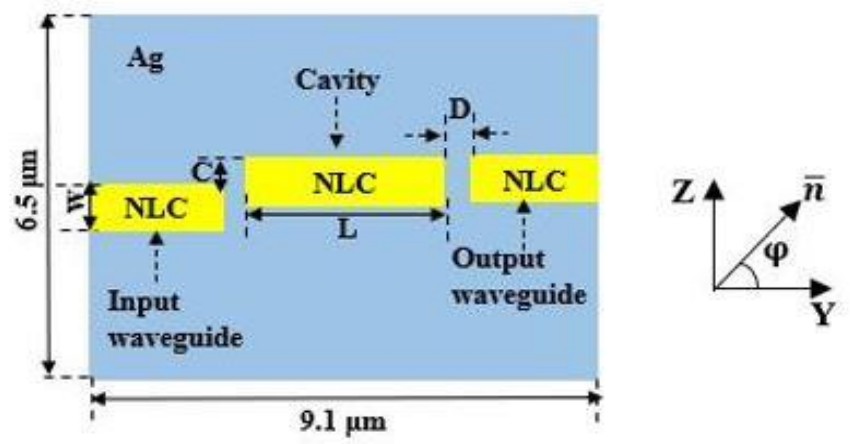

(a)

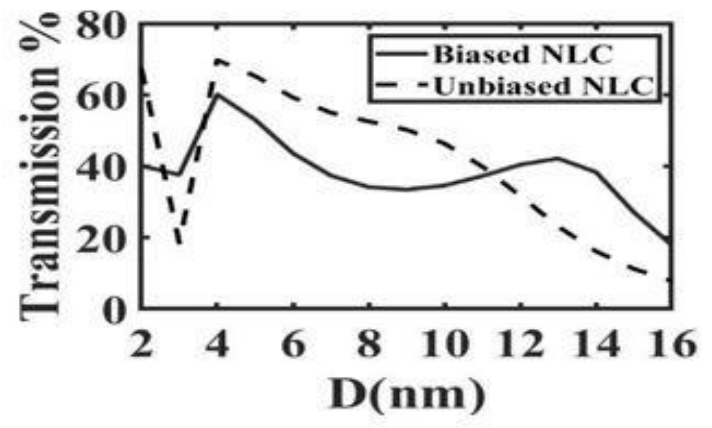

(b)

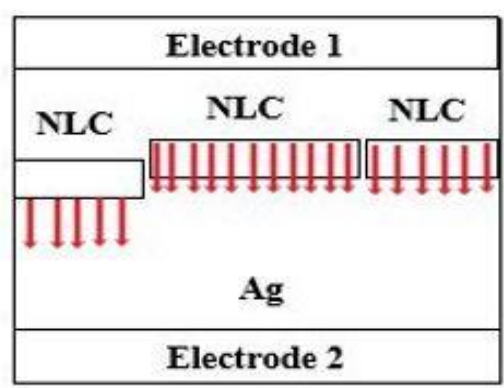

(c)

Fig. 2: (a) Tunable plasmonic mode converter using single output waveguide, the inset shows the director $\bar{n}$ of the NLC molecules with rotation angle $\varphi$ with respect to y-axis, (b) The transmission conversion efficiency at different values for D at biased and unbiased NLC states and (c) Electric field distribution along the NLC layers at the biased state.

In this study, the transmission conversion efficiency is defined as the ratio between the power at output and input ports. During the biased state of the NLC, the a- mode is coupled and converted to smode at the cavity waveguide. The output waveguide has transmission conversion efficiency of $35 \%$ which is the same as that reported by Wang and Yan (2018). In order to increase the transmission conversion efficiency, the dimensions of the structure are studied carefully. Figure 2 (b) shows the effect of the gap distance D on the transmission conversion efficiency at biased and unbiased cases. At the biased state, the a-mode will be converted to the s-mode while at the un-biased case; no mode conversion will occur. It is evident that high transmission efficiencies of $60 \%$ and $70 \%$ are achieved at $\mathrm{D}=4 \mathrm{~nm}$ for 
the biased and unbiased states, respectively which are higher than $35 \%$ that reported by Wang and Yan (2018). It should be noted that $4 \mathrm{~nm}$ separation distance has been experimentally achieved in (Lee et al. 2014).

Figure 3(a,b) show the calculated transmission conversion efficiency at different values of $\mathrm{w}$ and $\mathrm{C}$, respectively at the biased state of the NLC. It may be seen that high transmission conversion efficiency of $70 \%$ occurs at $\mathrm{C}=500 \mathrm{~nm}$ and $w=660 \mathrm{~nm}$. Figure 3(c, d) show the magnetic field propagation through the propagation plane $\mathrm{YZ}$ at different biased NLC states. It is evident from the figure that the obtained results are in a good agreement with the captured field distributions. At the unbiased state, the excited amode propagates through the design form the input to the output waveguide. However, at the biased NLC state, the input a-mode is converted to the s-mode at the output waveguide. The transmission ratio is equal to $70 \%$ for the s- mode at the biased state. The propagation losses and insertion losses are also calculated. In this study, the insertion losses (IL) is calculated according the following equation (Cheng et al. 2018)

$\mathrm{IL}=-10 \log (\mathrm{T})$

The a- mode will be converted to the s- mode during the biased state. The calculated propagation loss of the s-mode is equal to $20 \mathrm{~dB} / \mathbf{m m}$. Therefore, low propagation loss is achieved for the s- mode at a device length of $9.1 \mu \mathrm{m}$. Additionally, the insertion loss for the s- mode at the biased state is equal to $1.5 \mathrm{~dB}$.

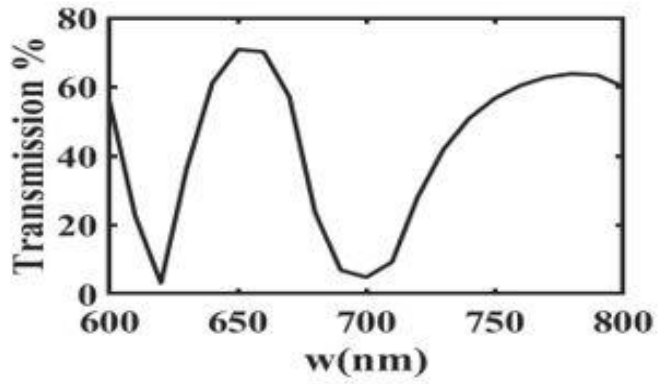

(a)

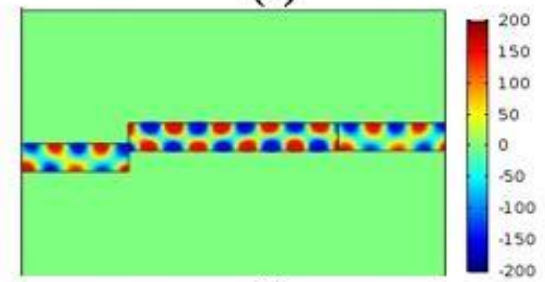

(c)

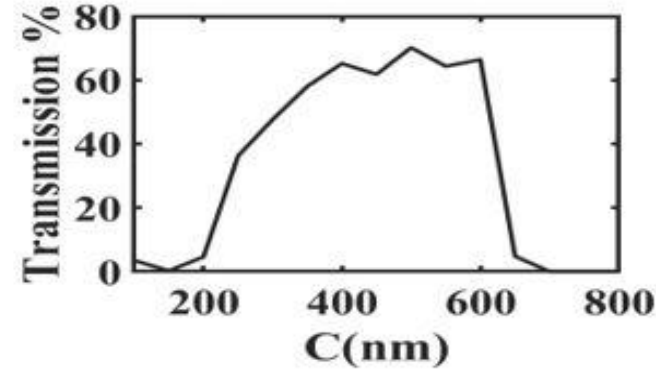

(b)

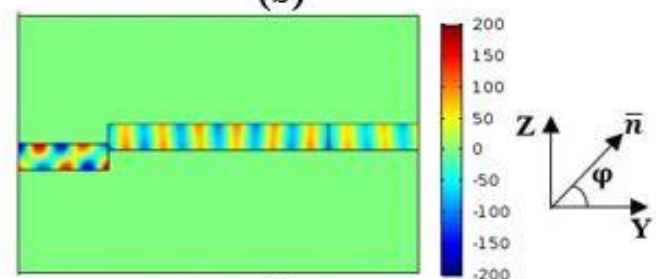

(d)

Fig. 3: Results of the switched plasmonic mode converter using single output waveguide: (a, b) Variation of transmission conversion efficiency at biased state with $\mathrm{w}$ and $\mathrm{C}$, respectively and the magnetic field distribution in the YZ propagation plane at (c) unbiased NLC and (d) biased NLC.

The effect of sharp corners of the cavity waveguide is also studied. Figure 4 shows the suggested design with rounded cavity and the transmission conversion efficiency at different radii of the rounded corners. In this study, the other geometrical parameters are used at their optimum values of $\mathrm{w}=660 \mathrm{~nm}$, $\underline{C}=500 \mathrm{~nm}$ and $\mathrm{D}=4 \mathrm{~nm}$. It is evident that the transmission is still better than $69 \%$ in the studied range from $\mathrm{r}=0$ to $50 \mathrm{~nm}$. It is worth noting that the supported modes will not be affected if the corners of the shifted cavity are rounded. 


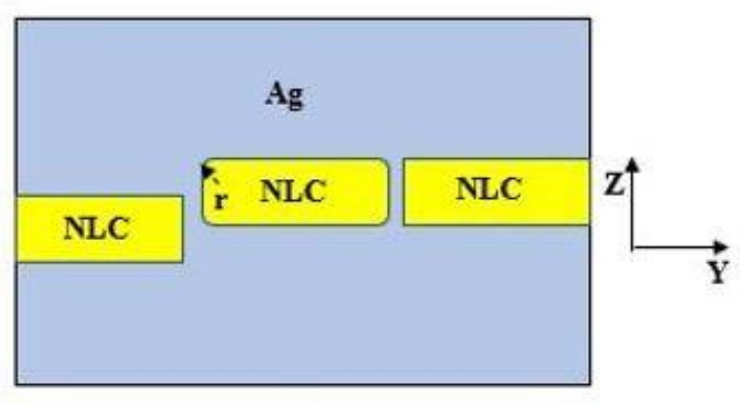

(a)

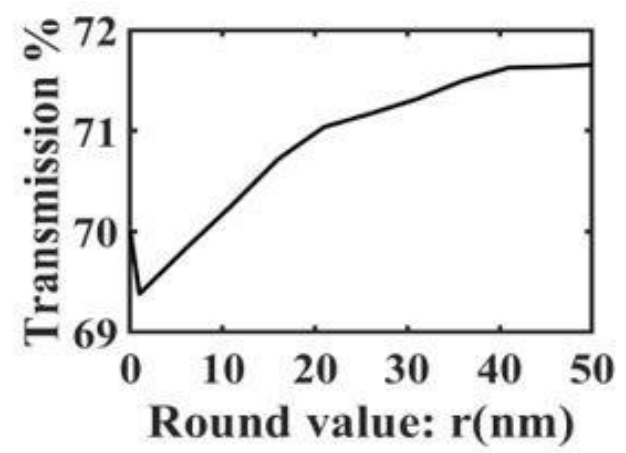

(b)

Fig. 4: (a) The proposed structure with rounded corners cavity and (b) The transmission conversion efficiency at different radii of the cavity corners.

\subsection{Tunable TM/TEM Converter Using Two Output Waveguides}

The tunable TM/ TEM converter is also suggested using two output waveguides configuration as shown in Fig. 5. The thickness of the added output waveguide is taken by $800 \mathrm{~nm}$ and is injected with NLC material. The distance between the output waveguides is adjusted carefully to generate the a-mode and smode simultaneously. In the current study, the input waveguide will be excited by the a-mode. At the biased state, the s- mode will be obtained from the upper waveguide while the a-mode will propagate through the lower waveguide. The situation will be reversed at the unbiased case where the a-mode will continue through the upper waveguide while the s-mode will be supported by the lower waveguide. The effect of the $\mathrm{D}$ distance on the transmission through the upper $\left(\mathrm{T}_{1}\right)$ and lower $\left(\mathrm{T}_{2}\right)$ waveguides at the biased state is shown in Fig. 5 (b). The other parameters are constant at B=60 nm, C $=500 \mathrm{~nm}$. It may be seen that during the biased NLC layer, the transmission conversion efficiency $\mathrm{T}_{1}$ of the s- mode is maximum at $\mathrm{D}=10 \mathrm{~nm}$. Moreover, it is worth noting that the distance $10 \mathrm{~nm}$ has been achieved as reported by Siegfried et al. (Siegfried et al. 2011). Figure 5 (c) and (d) show the captured magnetic field distribution at the unbiased and biased NLC layer, respectively. The results show that at the unbiased NLC layer, the two plasmonic modes are generated simultaneously, where the a-mode is excited in the upper output waveguide with transmission efficiency $49 \%$. However, the s-mode is generated in the second output waveguide with transmission conversion efficiency of $10 \%$. The situation is reversed at $\varphi$ $=90^{\circ}$. In this context, the a-mode is propagated in the lower output waveguide and the s-mode is excited in the upper output waveguide with transmission conversion efficiency of 50\%. The calculated propagation loss is equal to $17 \mathrm{~dB} / \mathrm{mm}$ for the s-mode. Therefore, low propagation loss is achieved for the s-mode at a device length of $9.1 \mu \mathrm{m}$ with a good transmission of 50\%. Additionally, the insertion loss for the s- mode at the biased state is equal to $3 \mathrm{~dB}$. The achieved results are better than that reported in Wang and Yan (2018) with small transmission of 35\%. In order to obtain equal transmission efficiency of $34 \%$ for both modes, $\mathrm{D}=7 \mathrm{~nm}$ should be used as shown in Fig. 5 (b). 


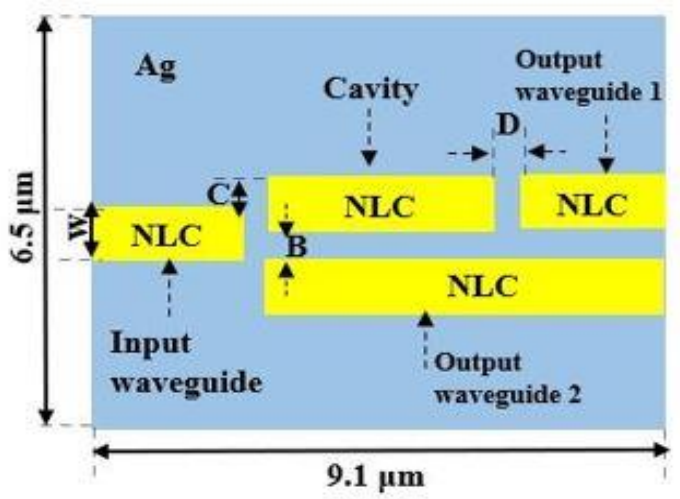

(a)

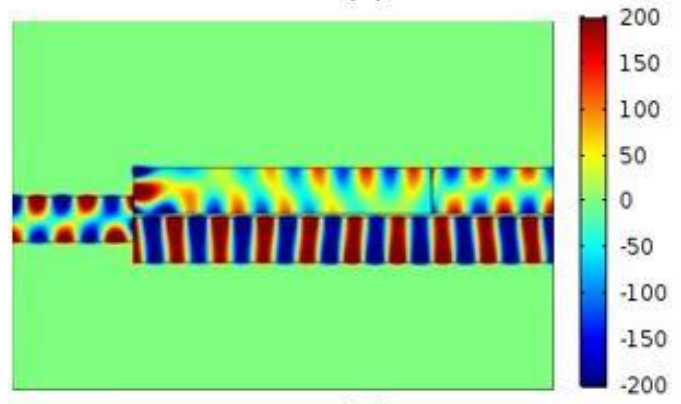

(c)

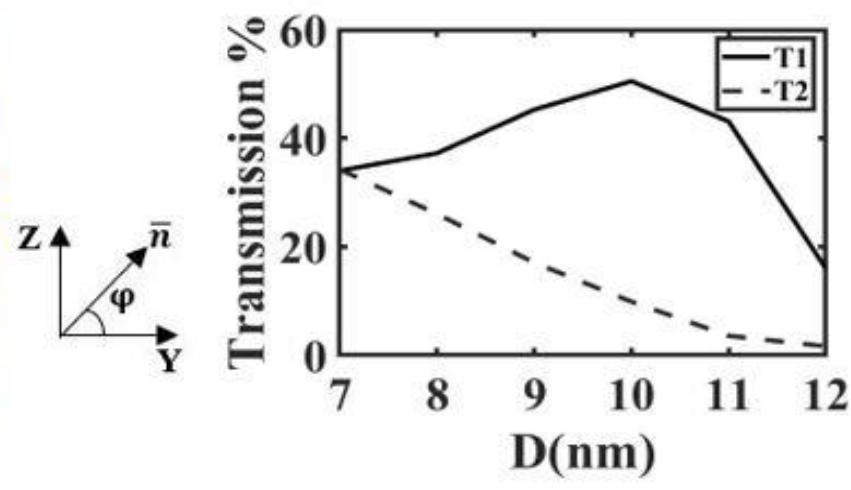

(b)

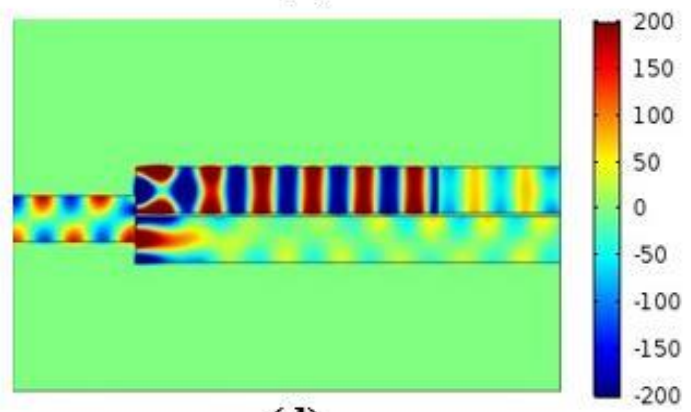

(d)

Fig. 5: (a) Tunable plasmonic mode converter using 2-output waveguides where the inset shows the director $\bar{n}$ of the NLC molecules with rotation angle $\varphi$ with respect to $y$ - axis, (b) the transmission conversion efficiency through the upper $\left(\mathrm{T}_{1}\right)$ and lower $\left(\mathrm{T}_{2}\right)$ waveguides at different values for D during the NLC biased state, and the magnetic field distribution in the YZ propagation plane for the (c) unbiased NLC $\left(\varphi=0^{\circ}\right)$, and (d) biased NLC $\left(\varphi=90^{\circ}\right)$.

The effect of the distance between the two waveguides $B$ is next studied at $D=10 \mathrm{~nm}$. It is found that at $\mathrm{B}=60 \mathrm{~nm}$, high transmission of $50 \%$ is achieved for the s-mode for the biased case. If $\mathrm{B}$ is varied, the transmission will be effectively reduced. Therefore, $B=60 \mathrm{~nm}$ will be chosen for the suggested design. The impact of the radius $r$ of the rounded corners on the transmission spectra are shown in Fig. 6 (b) at the biased state. It is evident that the transmission $\mathrm{T}_{1}$ of the s- mode is nearly constant at $50 \%$ in the studied range from $\mathrm{r}=0$ to $8 \mathrm{~nm}$ at the biased state. It is worth noting that the modes will not be affected if the corners of the shifted cavity are rounded

Table 1 shows a comparison between the proposed plasmonic mode configurations and the previously reported plasmonic mode converters. It is evident from the table that the suggested configuration has a small size and high transmission conversion efficiency compared to the previously reported structure suggested by Wang and Yan (2018). Additionally, thanks to the NLC, the proposed structure enables the excitation of the two plasmonic modes based on the biasing state of the NLC 


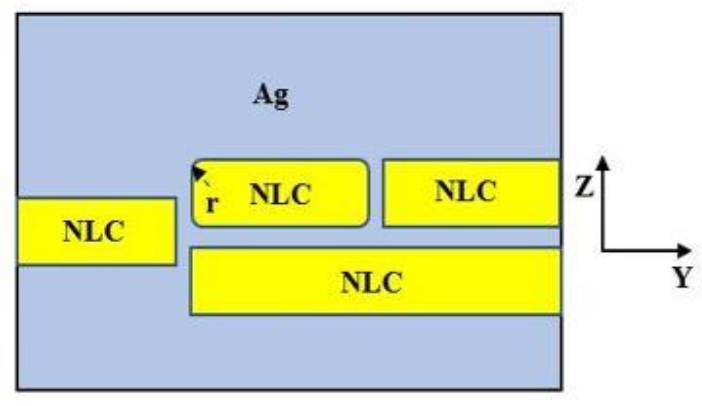

(a)

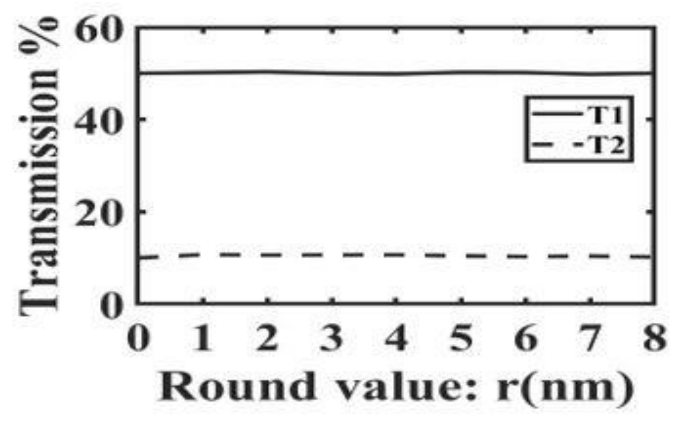

(b)

Fig. 6: (a) The proposed second structure with rounded corners cavity and (b) the transmission conversion efficiency of the s-mode $\left(\mathrm{T}_{1}\right)$ and a-mode $\left(\mathrm{T}_{2}\right)$ at different radii $\mathrm{r}$.

Table 1 comparison between the proposed plasmonic mode structure and the previously reported structures

\begin{tabular}{|c|c|c|c|c|c|}
\hline Structure & Materials & $\begin{array}{c}\text { Operating } \\
\text { Wavelength } \\
(\mathrm{nm})\end{array}$ & $\begin{array}{c}\text { Width } \\
\text { From input } \\
\text { to output } \\
(\mu \mathrm{m})\end{array}$ & $\begin{array}{c}\text { Transmission } \\
\text { Conversion } \\
\text { efficiency }\end{array}$ & $\underline{\text { Reconfigurability }}$ \\
\hline $\begin{array}{c}\text { (Ohana and Levy } \\
\text { 2014) }\end{array}$ & $\mathrm{Si}, \mathrm{SiO}_{2}$ & 1550 & 45 & $96.4 \%$ & No \\
\hline (Pan et al. 2014) & $\mathrm{Ag}, \mathrm{SiO}_{2}$ & 440 & $\mathrm{NA}$ & $\sim 40 \%$ & $\mathrm{No}$ \\
\hline $\begin{array}{c}\text { (Wang and Hong } \\
\text { 2017) }\end{array}$ & Air, Ag & 1310 & 12 & NA & No \\
\hline $\begin{array}{c}\text { (Wang and Yan } \\
\text { 2018) }\end{array}$ & Air, Ag & 1310 & 9 & $\sim 35 \%$ & No \\
\hline $\begin{array}{c}\text { Proposed Structure } \\
\text { using one output } \\
\text { waveguide }\end{array}$ & NLC, Ag & 1310 & 9.1 & $70 \%$ & Yes \\
\hline $\begin{array}{l}\text { Proposed Structure } \\
\text { using two output } \\
\text { waveguides }\end{array}$ & NLC, Ag & 1310 & 9.1 & $50 \%$ & Yes \\
\hline
\end{tabular}

\section{Conclusion}

In this paper, two compact and efficient tunable TM/TEM plasmonic mode converters are presented and simulated using COMSOL Multiphysics software package. The first configuration consists of silver layer with 3 rectangular holes injected by NLC layers. The operation is based on the excitation of the a- mode in the input waveguide that can be converted to s-mode in the output waveguide based on the biasing state of the NLC layer. The results show maximum conversion efficiency of $70 \%$. Additionally, in the second proposed configuration, two output MIM waveguides are suggested to allow the generation of the two plasmonic modes simultaneously. During the biased state, the s- mode transmission conversion efficiency reaches $50 \%$. The transmission of a- mode at the unbiased NLC layer is equal to $49 \%$. In comparison with 
the previously reported structures, the proposed configurations are characterized by small size, high transmission conversion efficiency and reconfigurability.

\section{References}

Areed, N. F., Hussien, M., \& Obayya, S. S.: Reconfigurable coupler-based metallic photonic crystal lens and nematic liquid crystal. JOSA B, 35(10), 2459-2466, (2018).

Borshch, V., Shiyanovskii, S. V., \& Lavrentovich, O. D.: Nanosecond electro-optic switching of a liquid crystal. Physical review letters, 111(10), 107802 (2013).

COMSOL Multiphysics Software; https://www.comsol.com.

Chen, Q., Wang, Y., \& Wu, Y.: Integer-programming model for plasmonic waveguide demultiplexers. Plasmonics, 10(2), 329-334, (2015).

Cheng, Z., Wang, J., Yang, Z., Yin, H., Wang, W., Huang, Y., \& Ren, X.: Broadband and high extinction ratio mode converter using the tapered hybrid plasmonic waveguide. IEEE Photonics Journal, 11(3), 1-8. (2019).

Dagens, B., Février, M., Gogol, P., Blaize, S., Apuzzo, A., Magno, G., ... \& Lérondel, G.: Direct observation of optical field phase carving in the vicinity of plasmonic metasurfaces. Nano letters, 16(7), $\underline{4014-4018(2016)}$.

Desmet, H., Neyts, K., \& Baets, R.: Liquid crystal orientation on patterns etched in Silicon on Insulator. In Integrated Optics, Silicon Photonics, and Photonic Integrated Circuits (Vol. 6183, p. 61831Z). International Society for Optics and Photonics. (2006, April).

Dionne, J. A., Sweatlock, L. A., Atwater, H. A., \& Polman, A.: Plasmon slot waveguides: Towards chipscale propagation with subwavelength-scale localization. Physical Review B, 73(3), 035407, (2006).

El-Rabiaey, M. A., Areed, N. F., \& Obayya, S. S.: Novel plasmonic data storage based on nematic liquid crystal layers. Journal of Lightwave Technology, 34(16), 3726-3732.(2016)

Eskalen, H., Özğan, Ș., ALVER, Ü., \& Kerli, S.: Electro-Optical Properties of Liquid Crystals Composite with Zinc Oxide Nanoparticles. Acta Physica Polonica, A., 127(3) (2015).

Fernández-Domínguez, A. I., Moreno, E., Martín-Moreno, L., \& García-Vidal, F. J.: Terahertz wedge plasmon polaritons. Optics letters, 34(13), 2063-2065, (2009).

Gramotnev, D. K., \& Bozhevolnyi, S. I.: Plasmonics beyond the diffraction limit. Nature photonics, 4(2), 83, (2010). 
Haakestad, M. W., Alkeskjold, T. T., Nielsen, M. D., Scolari, L., Riishede, J., Engan, H. E., \& Bjarklev, A.: Electrically tunable photonic bandgap guidance in a liquid-crystal-filled photonic crystal fiber. IEEE Photonics Technology Letters, 17(4), 819-821. (2005).

Hameed, M. F. O., \& Obayya, S. S. A.: Polarization rotator based on soft glass photonic crystal fiber with liquid crystal core. Journal of lightwave technology, 29(18), 2725-2731, (2011).

Hameed, M. F. O., Obayya, S. S. A., Wiltshire, R. J., Beam propagation analysis of polarization rotation in soft glass nematic liquid crystal photonic crystal fibers.: IEEE Photonics Technology Letters 22 (3), $188-190$

Hameed, M. F. O., Alrayk, Y.K. A., Shaalan, A. A., El Deeb, W. S., Obayya, S, S. A.: Design of highly sensitive multichannel bimetallic photonic crystal fiber biosensor, Journal of nanophotonics 10 (4), 046016, 2016

Hameed, M. F. O., Saadeldin, A. S., Elkaramany, E. M. A., Obayya, S. S. A., Label-free highly sensitive hybrid plasmonic biosensor for the detection of DNA hybridization, Journal of Lightwave Technology 35 (22), 4851-4858 (2017)

Hameed, M. F. O., \& Obayya, S. S.: Modal analysis of a novel soft glass photonic crystal fiber with liquid crystal core. Journal of lightwave technology, 30(1), 96-102. (2011).

Heikal, A. M., Hameed, M. F. O., Obayya, S. S. A.: Improved trenched channel plasmonic waveguide, Journal of lightwave technology 31 (13), 2184-2191 (2013)

Hung, Y. T., Huang, C. B., \& Huang, J. S.: Plasmonic mode converter for controlling optical impedance and nanoscale light-matter interaction. Optics express, 20(18), 20342-20355, (2012).

Johnson, P. B., \& Christy, R. W.: Optical constants of the noble metals. Physical review B, 6(12), 4370, (1972).

Kim, J., Lee, S. Y., Park, H., Lee, K., \& Lee, B.: Reflectionless compact plasmonic waveguide mode converter by using a mode-selective cavity. Optics express, 23(7), 9004-9013, (2015).

Kong, X. T., Li, Z. B., \& Tian, J. G.: Mode converter in metal-insulator-metal plasmonic waveguide designed by transformation optics. Optics express, 21(8), 9437-9446, (2013).

Krasavin, A. V., \& Zayats, A. V.: Guiding light at the nanoscale numerical optimization of ultrasubwavelength metallic wire plasmonic waveguides. Optics letters, 36(16), 3127-3129, (2011).

Lee, D. J., Yim, H. D., Lee, S. G., \& Beom- Hoan, O.: Tiny surface plasmon resonance sensor integrated on silicon waveguide based on vertical coupling into finite metal-insulator-metal plasmonic waveguide. Optics express, 19(21), 19895-19900, (2011). 
Lee, J., Song, J., Sung, G. Y., \& Shin, J. H. : Plasmonic Waveguide Ring Resonators with 4 nm Air Gap and $\lambda$ 02/15 000 Mode-Area Fabricated Using Photolithography. Nano letters, 14(10), 5533-5538, (2014).

Li, J., Wu, S. T., Brugioni, S., Meucci, R., \& Faetti, S.: Infrared refractive indices of liquid crystals. Journal of Applied Physics, 97(7), 073501. (2005).

Maier, S. A., Kik, P. G., \& Atwater, H. A.: Observation of coupled plasmon-polariton modes in Au nanoparticle chain waveguides of different lengths: Estimation of waveguide loss. Applied Physics Letters, 81(9), 1714-1716(2002).

Martz, J., Ferrini, R., Nüesch, F., Zuppiroli, L., Wild, B., Dunbar, L. A., ... \& Anand, S.: Liquid crystal infiltration of InP-based planar photonic crystals. Journal of applied physics, 99(10), 103105(2006).

Moreno, E., Garcia-Vidal, F. J., Rodrigo, S. G., Martin-Moreno, L., \& Bozhevolnyi, S. I.: Channel plasmon-polaritons modal shape, dispersion, and losses. Optics letters, 31(23), 3447-3449, (2006).

Obayya, S., Areed, N. F. F., Hameed, M. F. O., M. H. Abdelrazik, Optical nano-antennas for energy harvesting, Innovative Materials and Systems for Energy Harvesting Applications, 26-62 (2015)

Ohana, D., \& Levy, U.: Mode conversion based on dielectric metamaterial in silicon. Optics express, 22(22), 27617-27631, (2014).

Pan, D., Wei, H., Jia, Z., \& Xu, H.: Mode conversion of propagating surface plasmons in nanophotonic networks induced by structural symmetry breaking. Scientific reports, 4, 4993, (2014).

Ren, G., Shum, P., Yu, X., Hu, J., Wang, G., \& Gong, Y.:Polarization dependent guiding in liquid crystal filled photonic crystal fibers. Optics communications, 281(6), 1598-1606. (2008).

Siegfried, T., Ekinci, Y., Solak, H. H., Martin, O. J., \& Sigg, H.: Fabrication of sub-10 nm gap arrays over large areas for plasmonic sensors. Applied Physics Letters, 99(26), 280. (2011).

Sun, S., Chen, H. T., Zheng, W. J., \& Guo, G. Y.: Dispersion relation, propagation length and mode conversion of surface plasmon polaritons in silver double-nanowire systems. Optics express, 21(12), 14591-14605, (2013).

Tao, J., Wang, Q. J., \& Huang, X. G.: All-optical plasmonic switches based on coupled nano-disk cavity structures containing nonlinear material. Plasmonics, 6(4), 753, (2011).

Veronis, G., \& Fan, S.: Bends and splitters in metal-dielectric-metal subwavelength plasmonic waveguides. Applied physics letters, 87(13), 131102, (2005).

Wang, Y., \& Hong, X.: Mode Conversion of Metal-Insulator-Metal Waveguide with Gradient Thickness Medium. IEEE Photonics Technology Letters, 29(17), 1443-1446, (2017). 
Wang, Y., \& Yan, X.: Mode conversion in metal-insulator-metal waveguide with a shifted cavity. Japanese Journal of Applied Physics, 57(1), 010303, (2018).

Wei, L., Alkeskjold, T. T., \& Bjarklev, A.: Compact design of an electrically tunable and rotatable polarizer based on a liquid crystal photonic bandgap fiber. IEEE photonics technology letters, 21(21), 1633-1635. (2009).

Wen, K., Yan, L., Pan, W., Luo, B., Guo, Z., \& Guo, Y.: Wavelength demultiplexing structure based on a plasmonic metal-insulator-metal waveguide. Journal of Optics, 14(7), 075001, (2012).

Wolinski, T. R., Szaniawska, K., Bondarczuk, K., Lesiak, P., Domanski, A. W., Dabrowski, R., ... \& Wojcik, J.: Propagation properties of photonic crystal fibers filled with nematic liquid crystals. Optoelectronics Review, 13(2), 177. (2005).

Zayats, A. V., Smolyaninov, I. I., \& Maradudin, A. A.: Nano-optics of surface plasmon polaritons. Physics reports, 408(3-4), 131-314, (2005). 


\section{Figures}

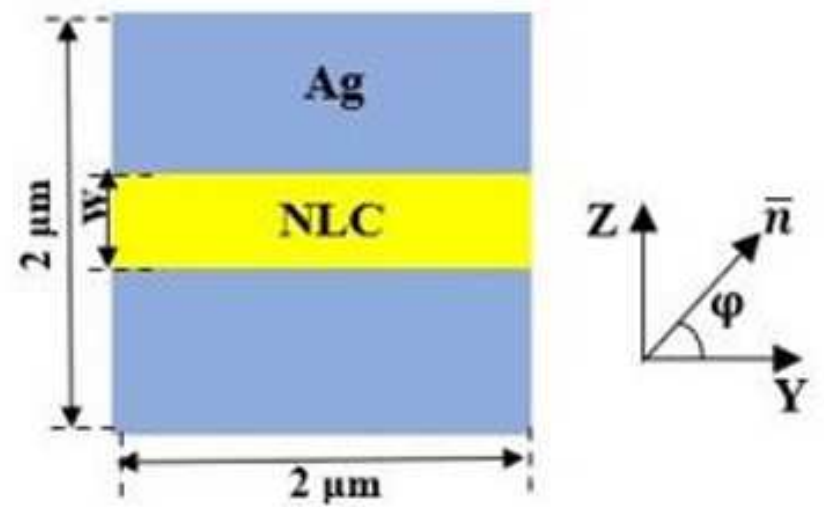

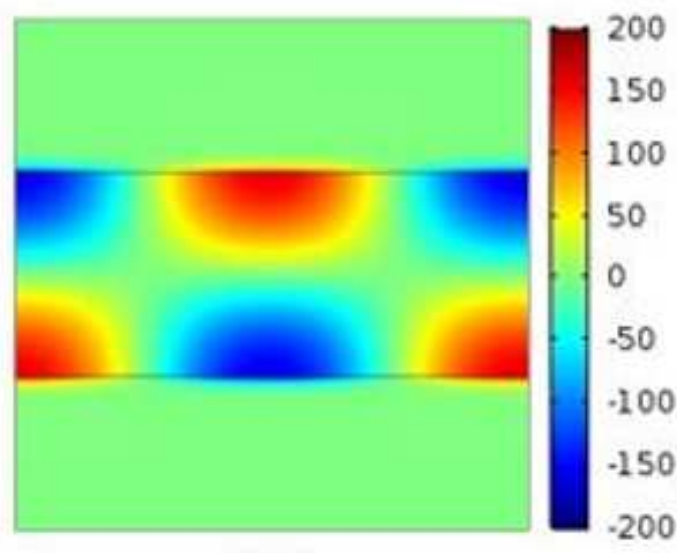

(b)

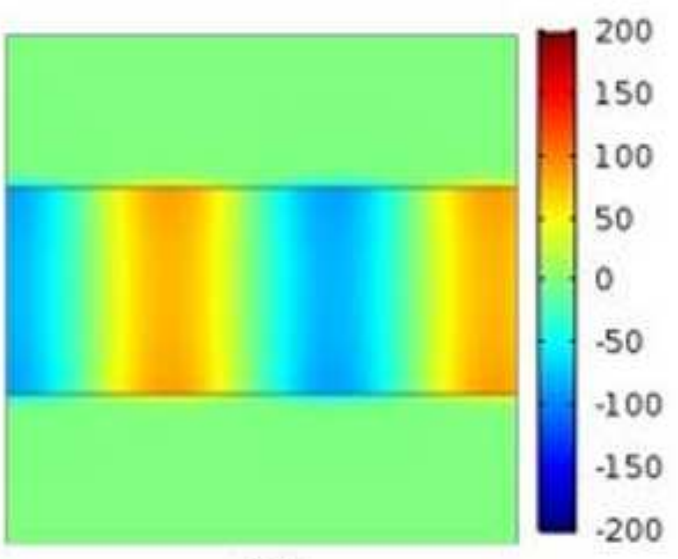

(c)

\section{Figure 1}

(a) The conventional MIM waveguide, and the magnetic field distributions in the YZ propagation plane of the (b) a-mode (TM mode), and (c) s-mode (TEM mode). 

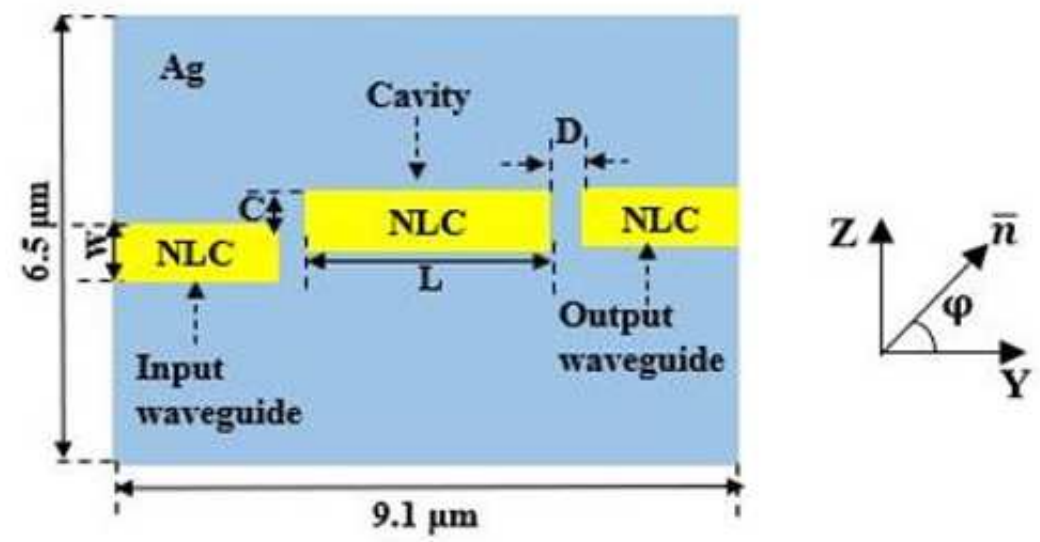

(a)

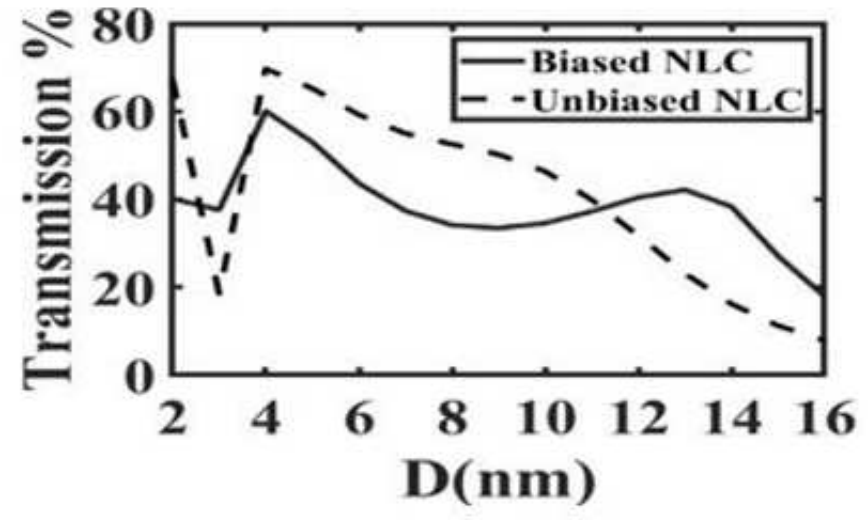

(b)

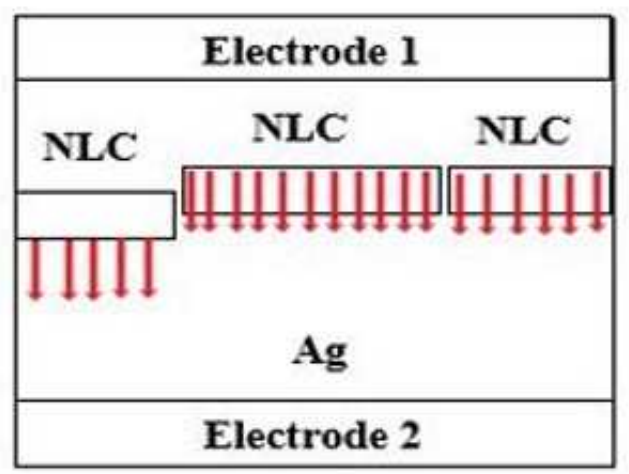

(c)

Figure 2

(a) Tunable plasmonic mode converter using single output waveguide, the inset shows the director $\mathrm{n} \otimes$ of

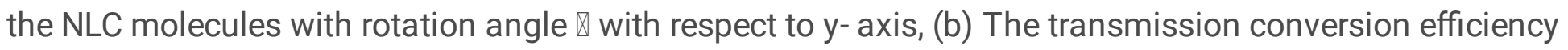
at different values for D at biased and unbiased NLC states and (c) Electric field distribution along the NLC layers at the biased state. 


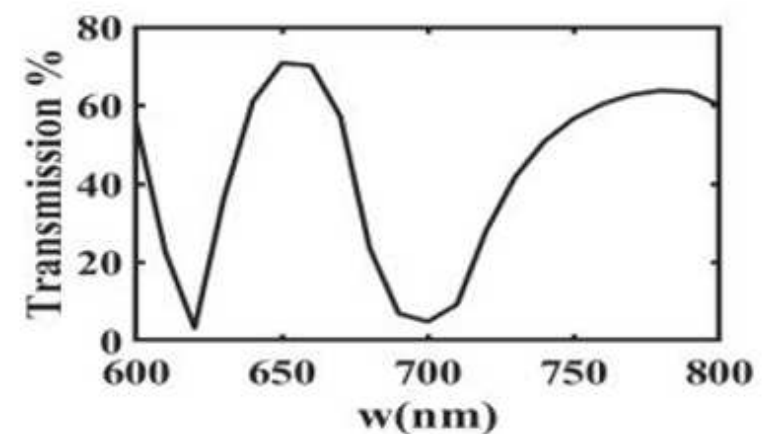

(a)

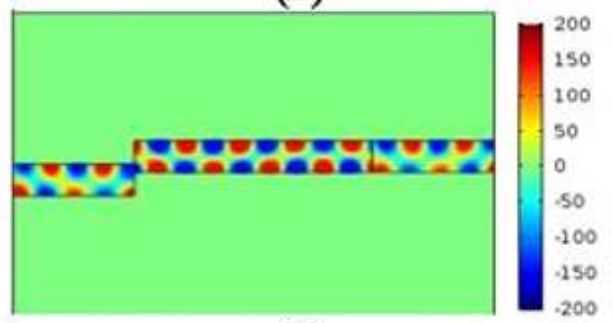

(c)

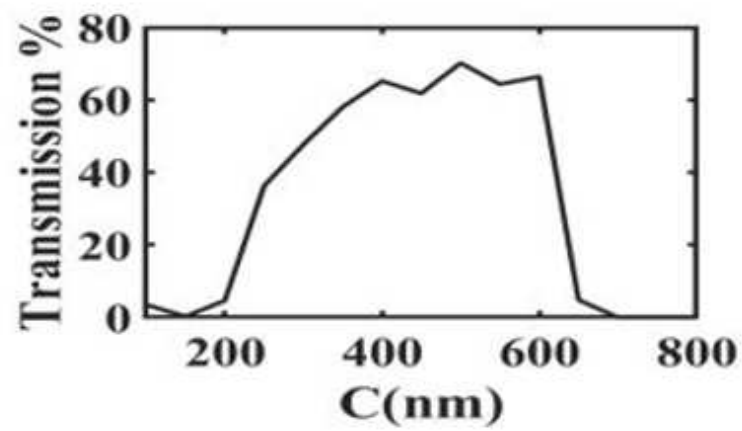

(b)

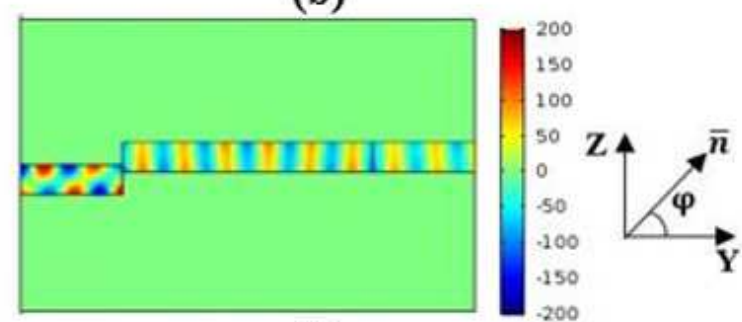

(d)

\section{Figure 3}

Results of the switched plasmonic mode converter using single output waveguide: $(a, b)$ Variation of transmission conversion efficiency at biased state with $\mathrm{W}$ and $\mathrm{C}$, respectively and the magnetic field distribution in the YZ propagation plane at (c) unbiased NLC and (d) biased NLC.

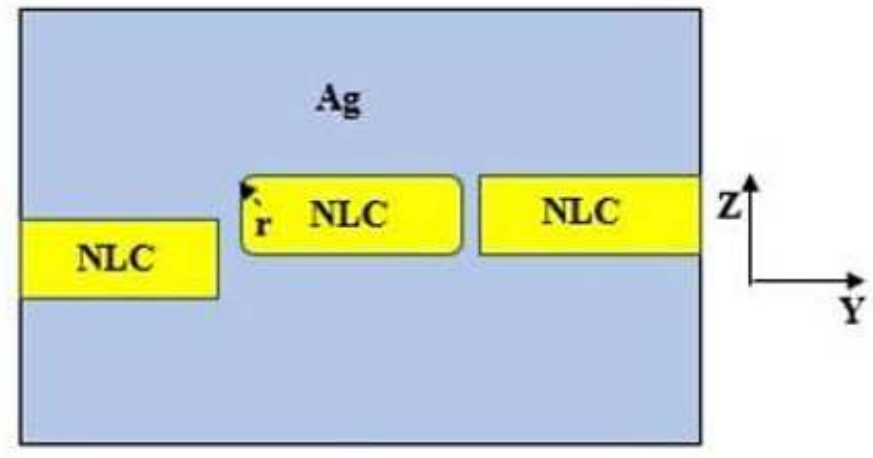

(a)

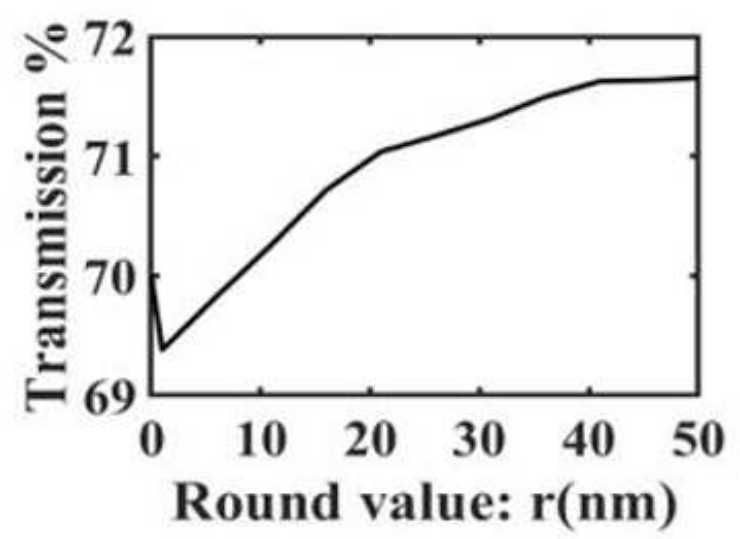

(b)

Figure 4

(a) The proposed structure with rounded corners cavity and (b) The transmission conversion efficiency at different radii of the cavity corners. 


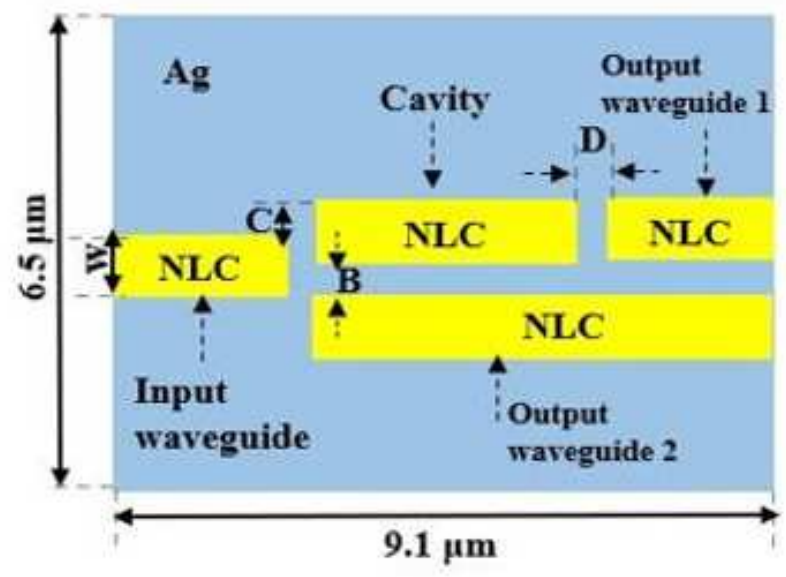

(a)

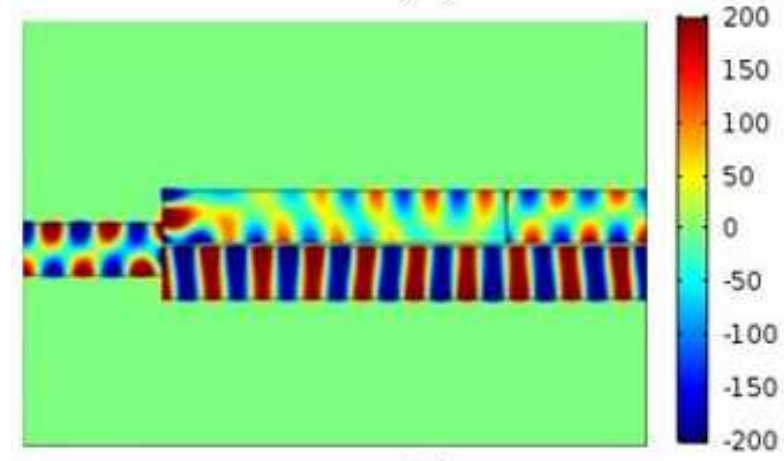

(c)

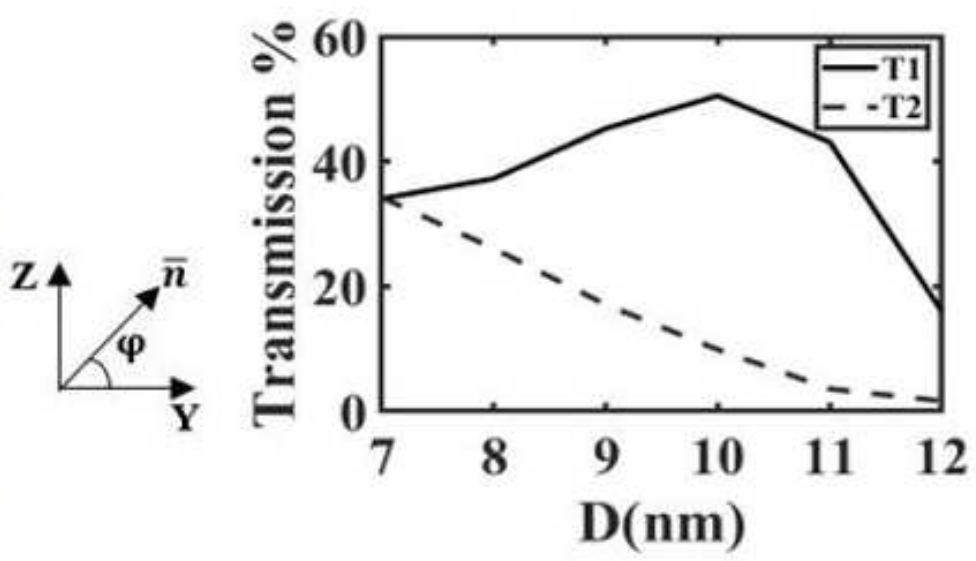

(b)

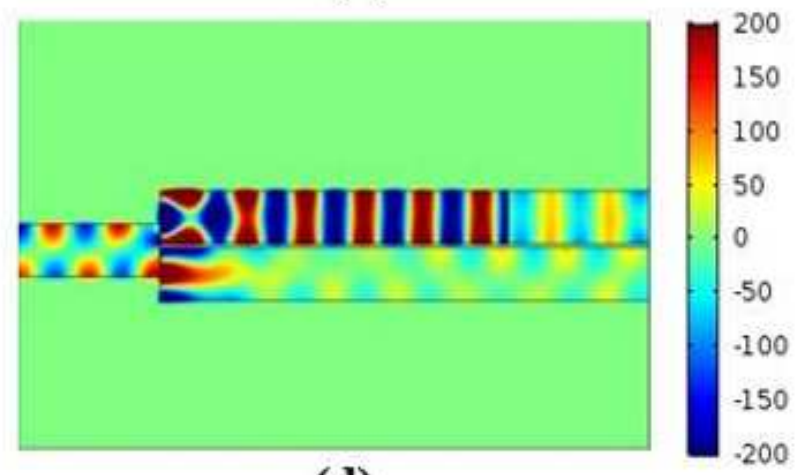

(d)

Figure 5

(a) Tunable plasmonic mode converter using 2-output waveguides where the inset shows the director $\mathrm{n} \rrbracket$

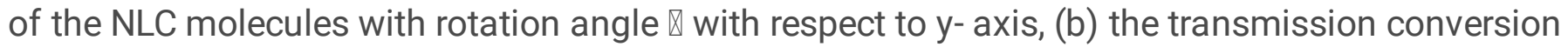
efficiency through the upper (T1) and lower (T2) waveguides at different values for D during the NLC biased state, and the magnetic field distribution in the YZ propagation plane for the (c) unbiased NLC ( $\varphi$ $=00)$, and $(\mathrm{d})$ biased NLC $(\varphi=900)$.

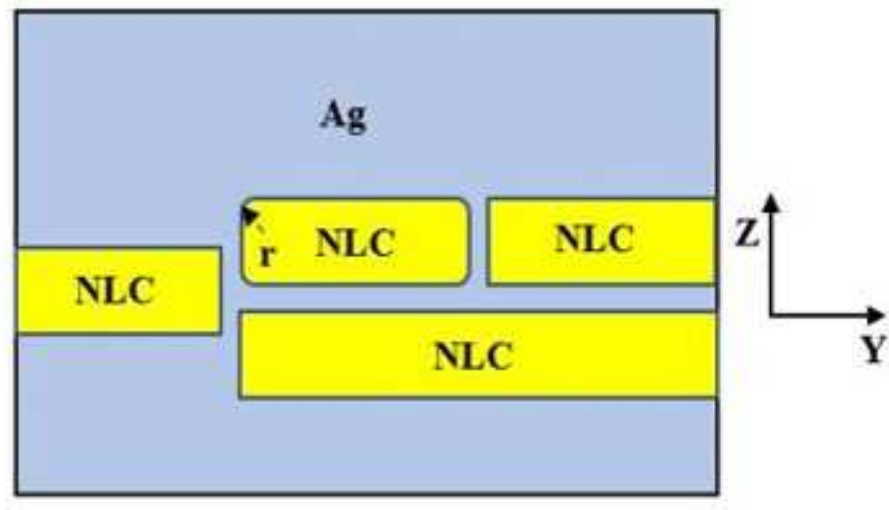

(a)

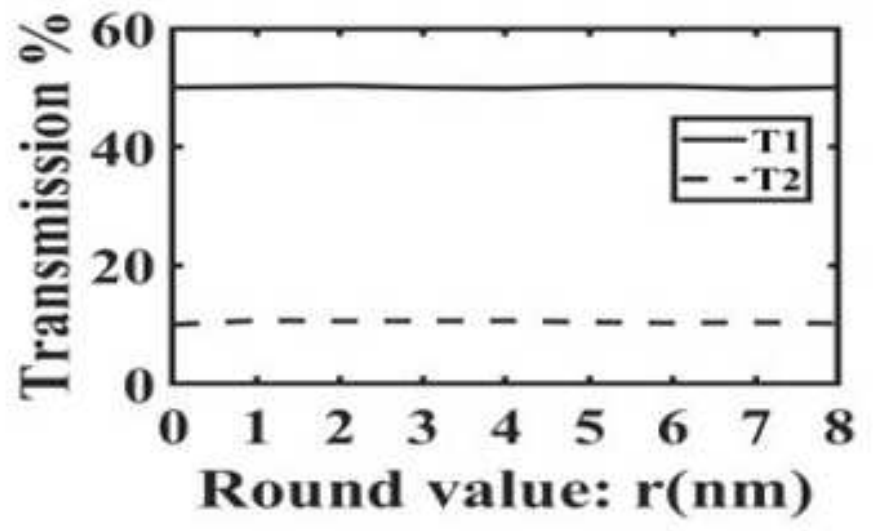

(b)

Figure 6 
Fig. 6: (a) The proposed second structure with rounded corners cavity and (b) the transmission conversion efficiency of the s-mode (T1) and a-mode (T2) at different radii r. 